\title{
The EU-ICARUS project: developing assistive robotic tools for search and rescue operations
}

\author{
Geert De Cubber, \\ Daniela Doroftei \\ Royal Military Academy \\ Brussels, Belgium \\ geert.de.cubber \\ @ rma.ac.be
}

\author{
Daniel Serrano \\ ASCAMM \\ Barcelona, Spain \\ dserrano \\ @ascamm.com
}

\author{
Keshav Chintamani \\ Space Applications Services \\ Zaventem, Belgium \\ keshav.chintamani \\ @ spaceapplications.com
}

\author{
Rui Sabino
ESRI Portugal
Lisboa, Portugal
rui.sabino
@esri-portugal.pt

Stephane Ourevitch

\author{
SpaceTec Partners \\ Brussels, Belgium \\ ourevitch \\ @ spacetecpartners.eu
}

\begin{abstract}
The ICARUS EU-FP7 project deals with the development of a set of integrated components to assist search and rescue teams in dealing with the difficult and dangerous, but lifesaving task of finding human survivors. The ICARUS tools consist of assistive unmanned air, ground and sea vehicles, equipped with victim detection sensors. The unmanned vehicles collaborate as a coordinated team, communicating via ad-hoc cognitive radio networking. To ensure optimal human-robot collaboration, these tools are seamlessly integrated into the C4I (command, control, communications, computers, and intelligence) equipment of the human crisis managers and a set of training and support tools is provided to them to learn to use the ICARUS system.
\end{abstract}

\section{INTRODUCTION}

Recent dramatic events such as the earthquakes in Japan and Haiti or the flooding in Pakistan have shown that emergency services often have difficulties with adequately managing crises. The result is that these crises lead to major disruption of the whole local society. The goal of ICARUS is to decrease the total cost of a major crisis. In order to attain this goal, the ICARUS project proposes to equip first responders with a comprehensive and integrated set of unmanned search and rescue (SAR) tools, to increase the situation awareness of human crisis managers, such that more rescue work can be done in a shorter amount of time.

In the event of large crises, a primordial task of the rescue services is the search for human survivors on the incident site. This is a complex and dangerous task, which often leads to loss of lives among the human crisis managers themselves. The introduction of unmanned SAR devices can offer a valuable tool to save human lives and to speed up the SAR process. ICARUS concentrates on the development of unmanned SAR technologies for detecting, locating and rescuing humans. In this context, there have already been many research efforts towards the development of unmanned SAR tools (see here for an overview: [1]). This research effort stands in contrast to the practical reality in the field, where unmanned SAR tools have great difficulty finding their way to the endusers, due to a number of remaining bottlenecks [2] in the practical applicability of unmanned tools. The ICARUS project addresses these issues, aiming to bridge the gap between the research community and end-users, by developing a toolbox of integrated components for unmanned SAR. In the following sections, the eight main ICARUS objectives are defined.

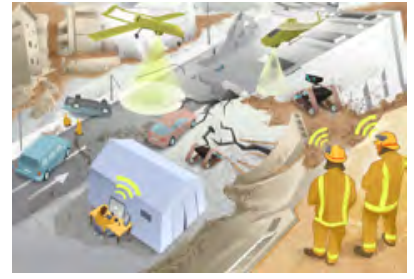

(a) Land Demonstration

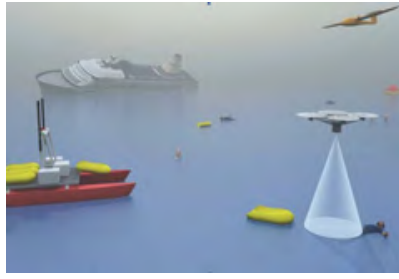

(b) Sea Demonstration
Fig. 1. ICARUS integrated demonstration scenarios

Real-life use case scenarios are foreseen for each objective, as defined by the end-users: Belgian First Aid and Support Team (B-FAST) and CINAV (Portuguese Navy). Two main scenarios are considered for demonstrating the integrated ICARUS results (see Fig. 1):

- An earthquake similar to the one in Haiti will be simulated at Marche-en-Famenne, Belgium. An integrated team of ICARUS UAS and UGVs will work in close collaboration with the B-FAST response team.

- A shipwreck similar to the Costa Concordia disaster will be simulated near Lisbon, Portugal. A team of unmanned surface vehicles and unmanned aerial vehicles will help the crisis managers in locating and providing immediate support to human survivors.

\section{DEVELOPMENT OF A LIGHT SENSOR CAPABLE OF DETECTING HUMANS}

A primary task for crisis management teams after the occurrence of a large disaster is the search for human survivors at the incident site. The objective of ICARUS is therefore to develop a small light-weight camera system capable of detecting human survivors. For human survivor detection, an infrared sensor with an ultra-high sensitivity in the mid-IR wavelength range seems the most adequate detection tool. Photovoltaic low-noise detectors such as the quantum cascade detector (QCD) [3] are very well suited to fulfill this requirement and will therefore be developed. These prototype cameras will have a resolution of $128 \times 128$ pixels arranged in a small array of $2 \times 2$ single chips. They will be based on novel and very promising QCD technology. The latter will allow the manufacture of highly sensitive, low noise, narrow band IR detectors with a detection wavelength of $8 \mu \mathrm{m}$. 
This ultra-sensitive, but relatively low-resolution QCD camera will be complemented by a commercial high-resolution lowersensitivity micro-bolometer camera. Minimal levels of weight $(500 \mathrm{~g})$, dimensions $(12 \times 12 \times 6 \mathrm{~cm})$ and total power consumption $(5 W)$ are being targeted. Image and video processing algorithms for detecting human survivors will be developed and combined to obtain sufficient detection performance. Data fusion methods will be applied to images coming from different cameras, resulting in different detection algorithms.

\section{DEVELOPMENT OF COOPERATIVE UNMANNED AERIAL SYSTEMS (UAS) FOR UNMANNED SAR}

End-users underline the important role Unmanned Aerial Systems could provide in SAR operations by providing continuous support to coordinators and operators in the field for mapping of topography and scenario [4]. This information is the basis for situation awareness and planning of both unmanned as well as manned missions. Next, UAS will be used for target observation. This allows an operator to quickly send a camera to a specific position and attitude as a remote eye-pair including tracking and following a moving target, e.g. for people search outdoors and indoors. The use of dedicated computer vision algorithms on-board the UAS will firstly allow for the localisation of bystanders/victims within an acceptable range of precision; and secondly for tracking them, then adapting the navigation and aid kit delivery [5]. Both features reduce workload as compared to existing manned search. This on-board capability is complementary to coordination and field teams as well as $\mathrm{C} 4 \mathrm{I}$ equipment that can process more accurate localisation information. Once localised, victims may not be quickly reachable by SAR teams due to distance, weather conditions and so forth. In this case, the UAS can deliver light first-aid kits, such as self-inflating emergency floatation devices to provide the victim with first emergency response. As a last mission type, UAS can act as communication relays when maintaining or deploying a ground communication network in remote areas is cumbersome or unfeasible. ICARUS UAS platforms will be given a crucial role by acting as quick deployment assets in the field to provide valuable information to enhance situation awareness in support of the assessment of crisis managers, as well as to enable tactical planning and decision-making. UAS platforms will be equipped with sensors tailored to SAR requirements, including the IR camera and victim detection algorithms, allowing for the localisation of victims. To meet the above demands, complementary platforms are proposed (see Fig. 2 and [4]):

- A small long-endurance solar aeroplane (3m wingspan) is meant to provide the highest view at a maximum height of $300 \mathrm{~m}$, and therefore enabling the mapping functionality and initial victim search. Payload other than small cameras is limited, but operation times span up to a day.

- A Quadrotor with a size of $1 \mathrm{~m}$ and a maximum payload of $1 \mathrm{~kg}$ will be used for delivery tasks outdoors and observation.

- A smaller Multicopter will be used for indoor people search. Consequently, on-board autonomous functionalities will be developed to decrease the operator workload and increase the operational efficiency in the overall C4I system.

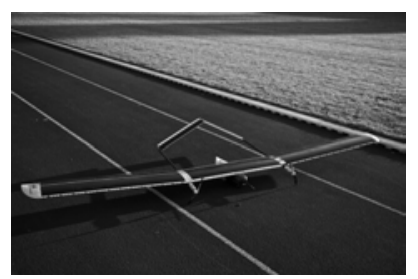

(a) Solar aeroplane by ETH

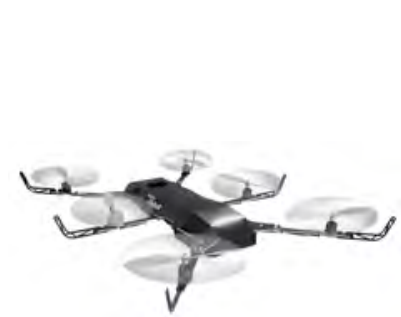

(c) Indoor multicopter by SkyBotix

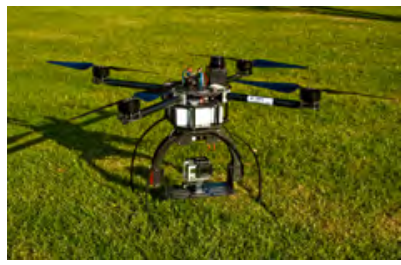

(b) Quadrotor by ASCAMM

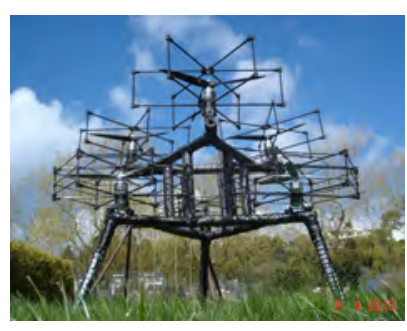

(d) Gyropendulum by JmdTheque
Fig. 2. UAS developed within the ICARUS project

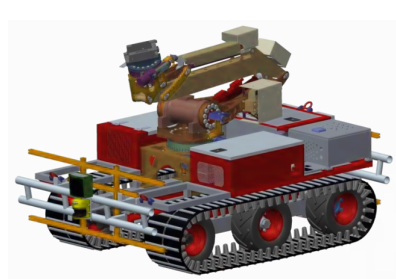

(a) Large UGV by Metalliance

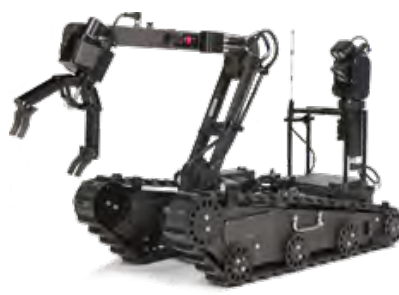

(b) Small UGV by Allen Vanguard
Fig. 3. UGVs developed within the ICARUS project

- An innovative Gyropendulum system, with a similar size to the Quadrotor, featuring more control authority, will be used for delivery in rough weather conditions in a semi-autonomous way.

\section{DEVElOPMENT OF COOPERATIVE UNMANNED GROUND VEHICLES (UGVS) FOR UNMANNED SAR}

End-users expressed the need for two types of robotic platforms in SAR operations [2]. In the first place, a large rough terrain - capable UGV (LUGV) is required which can be used as a mobile base, equipped with ample sensing capabilities, broadcasting the data it collects towards the field operators, as such increasing their situation awareness. The LUGV will have to carry several sensor systems and be powerful enough to surmount large obstacles. Therefore, it will be too large to drive into collapsed structures without the risk of causing even more damage or injuring victims. Hence a smaller UGV (SUGV) is also required, which will be able to enter in collapsed buildings to search for victims. The ICARUS project considers the development of such types of robotic systems [6], using existing base platforms (see Fig 3).

The LUGV shall move in a semi-autonomous way in a potentially hazardous and unknown environment. Within this context, it will act as a mobile sensor platform. Gathering a large amount of precise data is necessary for (semi-) autonomous navigation in challenging environments [7] as well as for the support of emergency teams. The LUGV shall be equipped with different sensor systems in order to account for 
the large variety of environment types the vehicle will possibly encounter. Next to its mission as a mobile sensor platform, the LUGV will also serve as a platform for a powerful manipulator that can be used to clear the vehicles path from obstacles or lift objects if a victim is buried beneath. The manipulator shall also be used to deploy the SUGV on the ground or even on higher structures such as the first floor of a collapsed building. The LUGV will be moved by a chain-drive for maximum manoeuvrability on difficult terrain. For localisation purposes, the platform will use an IMU and a satellite-based localisation system. The external sensor systems with which the LUGV will be equipped comprise two bumpers at the front and the rear of the system, a panning laser system for near-range terrain analysis that is also able to detect obstacles close-up in front of the robot and a stereo system. The victim detection sensors will be mounted on the platform. The manipulator will be controlled by an exoskeleton [8] and will be designed as 6-axis robotic arm that can lift up to approximately $250 \mathrm{~kg}$. Different tools will be provided and carried along on the LUGV.

The SUGV will be equipped with a propulsion system allowing it to manoeuvre in highly unstructured environments like collapsed buildings. Due to restrictions of size and weight, the autonomous capabilities will be more limited. A camera will be mounted on the SUGV, so a tele-operator can use it to gather information about the site of the disaster.

\section{DEVELOPMENT OF COOPERATIVE UNMANNED MARITIME SYSTEMS (UMS) FOR UNMANNED SAR}

Aquatic SAR operations face natural challenges since survival times of people in water are short, even in temperate climates. Furthermore, the risk for SAR teams has to be taken into account when deploying assets. Examples are accidents occurring during the night, under low visibility conditions, or under severe atmospheric or sea conditions, for which a fast response might put the SAR teams in great danger. For such operations, unmanned surface vehicles, capable of transporting search equipment and deploying first assistance devices, can reduce the arrival time at the incident area of basic life support equipment. At the same time, traditional life rafts that provide survivors with floatation can be robotised so that they can move autonomously and get close to survivors in the water, therefore reducing recovery time. This project proposes two main lines of work in order to address the identified demands. On one hand the project will present the instrumentation of a survival capsule to allow its motion towards survivors at the surface. On another hand the project will undertake the adaptation of a medium size USV (Unmanned Surface Vehicle) [9] for SAR operations, see Fig 4. Existing survival capsules that usually inflate when deployed allow survivors to climb aboard. The incorporation of power generation capabilities, a minimal set of instruments, basic communication equipment, and motion capabilities on board these capsules, will increase the lifesaving capabilities of such devices allowing their use in scenarios with reduced accessibility for other SAR services. USVs, as unmanned systems, allow remote human intervention under severe environmental conditions without putting additional people at risk. They have, therefore, a large potential for SAR operations at sea, especially under bad weather conditions with low visibility. Here, the adaptation of USVs for SAR will be pursued along the following lines:

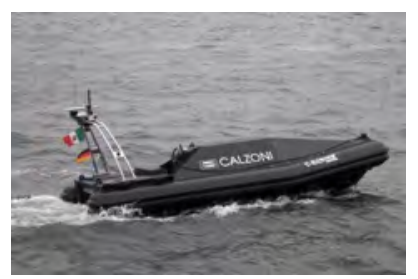

(a) U-Ranger by Calzoni

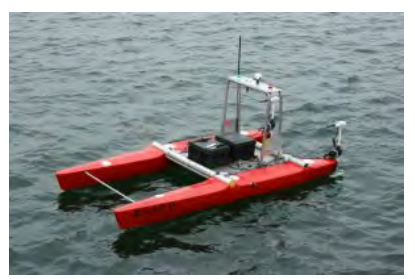

(b) ROAZ II by INESC
Fig. 4. UGVs developed within the ICARUS project

- $\quad$ Sensing and perception. Gathering data from different sensors installed on board the USV or from external data sources and combining it for target detection and tracking.

- Mission planning and control. Mission planning for operations with single or multiple vehicles, including obstacle avoidance manoeuvres.

- Capsule deployment system. Provide the USV with the capability of transporting and deploying lifesaving rafts in the incident area.

\section{HeTERogeneous ROBOT COLLABORATION BETWEEN UNMANNED SAR TOOLS}

The main concepts of interest for the end-users include the applicability of a Network Centric Operations paradigm, and compliance and compatibility with existing C4I systems. This objective is focused on a key enabling technology concept for the safe integration of autonomous platforms into SAR operations: the heterogeneous network. In this sense, the project specifically addresses the intrinsic capabilities and characteristics of a given platform, and how these characteristics are communicated, understood, and exploited by the SAR system.

In one sense, the heterogeneous network integration and management forms a central layer, with low-level control issues of specific vehicle types beneath it and mission-level planning, coordination and supervision at a higher layer. The present objective therefore addresses the integration of heterogeneous teams into a single, unified, interoperable system through establishing and demonstrating the interactions and use cases of different vehicle types, e.g. SAR mission operations integrating UAS and UGVs in a SAR task where the UAS provides support to the UGVs. The application of SAR influences the definition and interactions of the network, and this objective addresses therefore the challenges of interoperability through the implementation of standardised interfaces.

\section{DEVELOPMENT OF A SELF-ORGANISING COGNITIVE WIRELESS COMMUNICATION NETWORK}

SAR operations demand proper communication assets to ensure a highly available, real time networking capability for human and robotic teams working in SAR situations with hostile operational conditions. Key requirements are mobile and wireless communication capability for all of the involved entities with minimum deployment and coordination effort, individual and group communications with guaranteed quality and prioritisation capability and high capacity and range, security and power efficiency. No single technology currently supports all of the requirements at the same time. Thus, several 
technologies must be used simultaneously, which brings in a new and key requirement: interoperability. ICARUS will develop a network infrastructure which adapts to and takes advantage of the peculiarities of the posed SAR scenarios. A holistic approach will be followed, reusing state-of-the art solutions and focusing on:

- Mobile and wireless ad-hoc communications in mixed environments with robotic \& human actors, supporting both Line-of-Sight (LOS) \& non-LOS scenarios.

- Self-coordination and optimisation of spectrum resources by using cross-layer cognitive radio, maximising network usability and minimising interferences.

- $\quad$ Self-managed network able to adapt to varying conditions by using power-efficient, failure-resilient protocols and guidance of robotic network nodes.

- Flexible security scheme providing granular encryption, integrity and authentication.

- A harmonised management and control overlay on top of a highly robust waveform, able to encompass several data-link technologies ensuring interoperability.

\section{INTEGRATION OF UNMANNED SAR TOOLS IN THE COMMAND AND CONTROL SYSTEMS OF HUMAN SAR} FORCES

ICARUS aims at developing (robot) platform independent monitoring and control capabilities that will be able to handle, process and integrate a wide variety of data flows coming from sources such as the robotic platforms sensors, human beings (bystanders) in the field, GIS displaying a priori knowledge about the intervention field, etc. The resulting information and knowledge will primarily be exploited at the command and control application level, in order to effectively provide human operators with a high level of awareness allowing them to lead the robotic activities in a coordinated way with humans on field activities. As a noticeable feature, the command and control centre will provide a haptic tele-presence workstation allowing real-time control of haptic compliant robotic arms. This interface will in practice be demonstrated with a medium size hydraulic arm mounted on a UGV platform; with suitable control interfaces [10]. The command and control application [8] will allow the monitoring and control of heterogeneous robotic platforms including UAS, UGVs and UMS. The command and control will be designed to promote interoperability of the controlled systems, as well as aiming for seamless integration into existing infrastructure and applications used by first responders. The command and control development will come as an adaptation of, and integration with, a number of complementary existing technologies that partners will make available for the purpose of the project.

\section{DEVELOPMENT OF A TRAINING AND SUPPORT SYSTEM FOR THE UNMANNED SAR TOOLS}

Technological tools are no good for the human crisis managers if they do not know how to handle them. Therefore, an extensive training and support infrastructure is required. An important trend consists of designing trainers-simulators of the PC-type, and enabling e-training with the use of the Internet [11]. In the case of technological tools for SAR, such an infrastructure does not exist and is urgently required. In the ICARUS project several types of unmanned vehicles will be used, so from a training point of view the main objective is to deliver software tools that can simulate such a system. Different types of simulation (ground, air, water) will be developed and integrated to perform complex training of future ICARUS operators. The training tool will be capable of simulating predefined scenarios where virtual robots would send sensor data to the Command and Control Component operated by rescue services so that they can assess the simulated emergency and act accordingly. Furthermore, scenarios will be recorded from past events and then re-run for training purposes by using this tool. The Command and Control Component for support rescue services will integrate all sources of spatial information such as maps of the affected area, satellite images and sensor data coming from the unmanned robots in order to provide a situation snapshot to the rescue team and thus facilitate decision-making. An interactive human-machine interface that uses semantic information to operate robots will be used for rescue operations. The Command and Control Component will assign ICARUS robots to rescue teams, coordinating control decisions such that tasks can be executed with decreased risk.

\section{ACKNOWLEDGMENT}

The research leading to these results has received funding from the European Community's Seventh Framework Programme (FP7/2007-2013) under grant agreement n 285417.

\section{REFERENCES}

[1] G. Kruijff, F. Colas, T. Svoboda, J. van Diggelen, P. Balmer, F. Pirri, and R. Worst, "Designing intelligent robots for human-robot teaming in urban search \& rescue," in Proceedings of the AAAI 2012 Spring Symposium on Designing Intelligent Robots, 2012.

[2] D. Doroftei, G. De Cubber, and K. Chintamani, "Towards collaborative human and robotic rescue workers," in 5th International Workshop on Human-Friendly Robotics (HFR2012), October 2012.

[3] D. Hofstetter, F. Giorgetta, E. Baumann, Q. Yang, C. Manz, and K. Khler, "Mid-infrared quantum cascade detectors for applications inspectroscopy and pyrometry," Applied Physics B, vol. 100, no. 2, pp. 313-320, 2010.

[4] G. De Cubber, D. Doroftei, Y. Baudoin, D. Serrano, K. Chintamani, R. Sabino, and S. Ourevitch, "Operational rpas scenarios envisaged for search \& rescue by the eu fp7 icarus project," in Remotely Piloted Aircraft Systems for Civil Operations (RPAS2012), December 2012.

[5] S. Leutenegger and R. Siegwart, "A low-cost and fail-safe inertial navigation system for airplanes," in IEEE Int. Conf. on Robotics and Automation (ICRA), St. Paul, USA, May 2012, pp. 612-618.

[6] G. De Cubber, D. Doroftei, Y. Baudoin, D. Serrano, K. Berns, C. Armbrust, K. Chintamani, R. Sabino, and S. Ourevitch, "Search and rescue robots developed by the european icarus project," in 7th Int. Workshop on Robotics for Risky Environments, October 2013.

[7] K. Berns, K.-D. Kuhnert, and C. Armbrust, "Off-road roboticsan overview,” KI - Knstliche Intelligenz, vol. 25, no. 2, pp. 109-116, 2011.

[8] S. Govindaraj, K. Chintamani, J. Gancet, P. Letier, B. van Lierde, Y. Nevatia, G. De Cubber, D. Serrano, M. E. Palomares, J. Bedkowski, C. Armbrust, J. Sanchez, A. Coelho, and I. Orbe, "The icarus project - command, control and intelligence (c2i)," in IEEE International Symposium on Safety, Security, and Rescue Robotics, 2013.

[9] A. Martins, H. Ferreira, C. Almeida, H. Silva, J. M. Almeida, and E. Silva, "Roaz and roaz ii autonomous surface vehicle design and implementation," in Int. Lifesaving Congress, Spain, Dec. 2007.

[10] K. Chintamani, "Augmented reality navigation interfaces improve human performance in end-effector controlled telerobotics," Ph.D. dissertation, Wayne State University, 2010.

[11] J. Bedkowski, A. Maslowski, and G. De Cubber, "Real time 3d localization and mapping for usar robotic application," Industrial Robot: An International Journal, vol. 39, no. 5, pp. 464-474, 2012. 